\title{
Investigation of the Electrical Properties of Microtubule Ensembles under Cell-Like Conditions
}

\author{
Aarat P. Kalra ${ }^{1} ®$, Sahil D. Patel ${ }^{2}$, Asadullah F. Bhuiyan ${ }^{2}$, Jordane Preto ${ }^{1}$, Kyle G. Scheuer ${ }^{2}$,

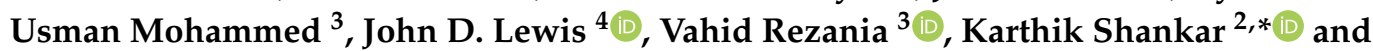 \\ Jack A. Tuszynski ${ }^{1,4}$ \\ 1 Department of Physics, University of Alberta, 11335 Saskatchewan Dr NW, Edmonton, AB T6G 2M9, \\ Canada; aarat@ualberta.ca (A.P.K.); preto@ualberta.ca (J.P.); jackt@ualberta.ca (J.A.T.) \\ 2 Department of Electrical and Computer Engineering, University of Alberta, 9107-116 St, Edmonton, \\ AB T6G 2V4, Canada; sdpatel@ualberta.ca (S.D.P.); abhuiyan@ualberta.ca (A.F.B.); \\ scheuer@ualberta.ca (K.G.S.) \\ 3 Department of Physical Sciences, MacEwan University, Edmonton, AB T5J 4S2, Canada; \\ mohammedu@mymacewan.ca (U.M.); rezaniav@macewan.ca (V.R.) \\ 4 Department of Oncology, University of Alberta, Edmonton, AB T6G 1Z2, Canada; jdlewis@ualberta.ca \\ * Correspondence: kshankar@ualberta.ca
}

Received: 4 December 2019; Accepted: 29 January 2020; Published: 5 February 2020

check for updates

\begin{abstract}
Microtubules are hollow cylindrical polymers composed of the highly negatively-charged ( 23e), high dipole moment (1750 D) protein $\alpha, \beta$-tubulin. While the roles of microtubules in chromosomal segregation, macromolecular transport, and cell migration are relatively well-understood, studies on the electrical properties of microtubules have only recently gained strong interest. Here, we show that while microtubules at physiological concentrations increase solution capacitance, free tubulin has no appreciable effect. Further, we observed a decrease in electrical resistance of solution, with charge transport peaking between $20-60 \mathrm{~Hz}$ in the presence of microtubules, consistent with recent findings that microtubules exhibit electric oscillations at such low frequencies. We were able to quantify the capacitance and resistance of the microtubules (MT) network at physiological tubulin concentrations to be $1.27 \times 10^{-5} \mathrm{~F}$ and $9.74 \times 10^{4} \Omega$. Our results show that in addition to macromolecular transport, microtubules also act as charge storage devices through counterionic condensation across a broad frequency spectrum. We conclude with a hypothesis of an electrically tunable cytoskeleton where the dielectric properties of tubulin are polymerisation-state dependent.
\end{abstract}

Keywords: microtubules; bioelectricity; bionanowires; neuronal charge storage; impedance spectroscopy; cytoskeleton

\section{Introduction}

Microtubules (MTs) are cylindrical polymers composed of the heterodimers of protein $\alpha, \beta-$ tubulin that play a variety of well-recognised intracellular roles, such as maintaining the shape and rigidity of the cell, aiding in positioning and stabilisation of the mitotic spindle for allowing chromosomal segregation, acting as 'rails' for macromolecular transport and forming cilia and flagella for cell movement. Since the tubulin dimer possesses a high negative electric charge of $\sim 23 \mathrm{e}$ and a large intrinsic high dipole moment of approximately $1750 \mathrm{D}$ [1,2], MTs have been implicated in electrically-mediated biological roles [3-6]. They have been modelled as nanowires capable of enhancing ionic transport [7,8], and simulated to receive and attenuate electrical oscillations [4,9-11]. In solution, MTs have been shown to align with applied electric fields [2,12-16]. Recently, MTs have 
also been modelled as the primary cellular targets for low-intensity (1-2 V), intermediate-frequency (100-300 kHz) electric fields termed TTFields (tumour-treating electric fields) that inhibit cancer cell proliferation, in particular glioma [17-19]. Indeed, MTs have been reported to decrease buffer solution resistance [12,13], leading to a conductance peak at frequencies close to the TTField regime [20]. While these studies show that MTs are highly sensitive to external electric fields, answers to the questions 'How do MTs effect a solution's capacitance?' and 'What is the capacitance of a single MT?' are still elusive and crucial to the determination of the dielectric properties of living cells. The tubulin concentration in mammalian cells varies in the micromolar range $(\sim 10-25 \mu \mathrm{M})[21,22]$. In vitro, polymerizing tubulin at such high concentrations can lead to the formation of entangled networks, confounding quantification of the individual MT response to electric fields. Electro-rotation, di-electrophoresis and impedance spectroscopy are thus performed using low concentrations of tubulin, in the nanomolar regime, to enable robust observation of individual MTs.

MT formation and stability are known to be optimal in buffers with ionic strength between 80 and $100 \mathrm{mM}[23,24]$. A background of BRB80 (which consists of $80 \mathrm{mM}$ PIPES, $2 \mathrm{mM} \mathrm{MgCl} 2$ and $0.5 \mathrm{mM}$ EGTA, containing $46 \mathrm{mM} \mathrm{PIPES}^{2-}$, $36 \mathrm{mM} \mathrm{PIPES}^{-}, \sim 68 \mathrm{mM} \mathrm{Cl}^{-}, \sim 160 \mathrm{mM} \mathrm{K}^{+}$, and $2 \mathrm{mM}$ $\mathrm{Mg}^{2+}$ [2]), is thus used to study the dynamics and mechanical properties of MTs. To study their electrical properties however, the usage of such high ionic-strength solutions has historically been problematic because any dielectric attenuation caused by MTs is overwhelmed by the noise and high conductivity from the background. In the low-frequency regime (1-100 kHz), two approaches have thus far been used to estimate the dielectric properties of MTs and tubulin. One is to electrically observe low concentrations of MTs (tubulin concentration in the nanomolar regime) in the presence of low ionic strengths $[12,13,20,25,26]$. Such studies overlook the intrinsic ionic concentration of mammalian cytosol, which varies between 200 to $500 \mathrm{mM}$ depending on the cell type [27,28]. Another approach to electrically interrogate MTs is to dry them: the conductivity of the buffer is nullified by evaporation, leaving polymeric tubulin behind $[29,30]$. In a physiological situation however, MTs are solvated by the highly conductive and noisy cytosol.

Here, we report on our efforts overcome the barrier posed by a high ionic strength by performing electrochemical impedance spectroscopy (EIS) on cellular concentrations of tubulin. We have been able to successfully observe differences in impedance using a background of BRB80 itself. Surprisingly, we find that MTs increase the solution capacitance of BRB80 whereas free tubulin does not, implicating a difference in electrical properties based only on the morphology of this protein solute. We also report a 'reversal' in the resistive behaviour of MTs compared to BRB80, with a reduction in solution resistance peaking in the 20-60 Hz region, a finding consistent with recent reports showing that polymerised tubulin quasi-resonantly responds to electric oscillations at $\sim 39 \mathrm{~Hz}[9,10]$. Using an equivalent circuit model for MTs, we experimentally determine the capacitance and resistance of the MT network to be $1.27 \times 10^{-5} \mathrm{~F}$ and $9.74 \times 10^{4} \Omega$ respectively, at physiological concentrations of tubulin. Our values indicate that the polymerisation of tubulin into MTs alters the spatial and temporal charge distribution, altering the electrical properties through charge storage in the cell.

\section{Materials and Methods}

\subsection{Tubulin Reconstitution}

Fluorescently labelled tubulin solution was prepared using previously published protocols [20]. Notably, tubulin stock powders that were devoid of MAPs were purchased. Lyophilised unlabelled tubulin powder (Cytoskeleton Inc, Denver, CO, USA; T240) was reconstituted in BRB80 supplemented with 1 mM GTP (guanosine triphosphate; Cytoskeleton Inc, Denver, CO, USA; BST06) and mixed with tubulin labelled with a rhodamine-based ester (Cytoskeleton Inc, Denver, CO, USA; TL590m) in a final labelling ratio of 1:15. Aliquots were snap-frozen and stored at $-80{ }^{\circ} \mathrm{C}$. 


\subsection{MT Polymerisation and Stabilisation}

MT polymerisation was performed by incubating $45.45 \mu \mathrm{M}$ tubulin aliquots in a $37^{\circ} \mathrm{C}$ water bath for 30 minutes. BRB80 solution was heated alongside tubulin during the first 15 minutes of polymerisation. Subsequently, BRB80 was incubated at room temperature, and paclitaxel solution (Cytoskeleton Inc, Denver, CO, USA; TXD01; 2 mM stock) was thawed at room temperature alongside it. After 30 minutes of tubulin polymerisation brought to completion, $100 \mu \mathrm{L}$ of BRB80 was added to $5 \mu \mathrm{L}$ of $2 \mathrm{mM}$ paclitaxel (BRB80T). For preparing $0.222,2.225$ and $22.225 \mu \mathrm{M}$ MTs the above solution was added in different volumes to polymerised tubulin, as shown in Table 1. For preparing the BRB80T background for impedance measurements, $45 \mu \mathrm{L}$ of this solution was added to $45 \mu \mathrm{L}$ of BRB80.

Table 1. Volumes of tubulin and buffer solution (BRB80T or BRB80C) used to stabilise microtubules (BRB80T) or free tubulin (BRB80C) in solution.

\begin{tabular}{ccc}
\hline Tubulin Concentration $(\mu \mathrm{M})$ & Volume of BRB80T or BRB80C $(\mu \mathrm{L})$ & Tubulin Volume $(\mu \mathrm{L})$ \\
\hline 0.222 & 99.5 & 0.5 \\
\hline 2.222 & 95 & 5 \\
\hline 22.225 & 5 & 5 \\
\hline
\end{tabular}

For tubulin stabilisation, $2 \mu \mathrm{L}$ of $5 \mathrm{mM}$ colchicine stock solution (Sigma-Aldrich, St. Louis, $\mathrm{MO}$, USA; C9754; $5 \mathrm{mM}$ in DMSO) was added to $100 \mu \mathrm{L}$ BRB80 (BRB80C). Subsequently, a similar solution to BRB80T was prepared using colchicine. For preparing $0.222,2.225$ and $22.225 \mu \mathrm{M}$ free tubulin solutions, the above solution was added in different volumes to free tubulin solutions, as shown in Table 1. For preparing BRB80C, $45 \mu \mathrm{L}$ of this solution was added to $45 \mu \mathrm{L}$ of BRB80.

\subsection{Fluorescence Imaging of MTs}

Imaging was performed on a Zeiss Examiner.Z1 microscope using a Hamamatsu (Hamamatsu City, Japan) EMCCD C9100 camera, a Zeiss (Oberkochen, Germany) plan-Apochromat 1.4 NA 63x lens. After pipetting MT solution (2-5 $\mu \mathrm{L}$ ) onto a glass slide (VWR 48382-173) a coverslip (VWR 48393-070) was placed on the solution, allowing it to spread. The microscope used an EXFO X-Cite 120 fluorescence source and excitation and emission filters of $535 \mathrm{~nm}$ and $610 \mathrm{~nm}$, respectively. Exposure times between $50 \mathrm{~ms}$ and $300 \mathrm{~ms}$ were used for imaging to validate the presence of MTs.

\subsection{Electrode Design and Device Construction}

Each 'plate' in the parallel-plate contact device was formed by FTO (fluorine-doped tin oxide)-coated glass slides (Sigma Aldrich, St. Louis, MO, USA; 735140). The slides were cleaved to dimensions of $1.5 \mathrm{~mm} \times 10 \mathrm{~mm} \times 50 \mathrm{~mm}$ for the upper contact and $1.5 \mathrm{~mm} \times 27 \mathrm{~mm} \times 50 \mathrm{~mm}$ for the lower contact. The cleaving dimensions were set using 3D printed devices that were placed as holders (The Shack, University of Alberta; Figure S1 in Supplementary Materials). The slides were ultrasonicated and subjected to reactive ion etching (RIE) using a 5-minute exposure to oxygen plasma (Oxford Instruments, Abingdon, UK; NGP80) to remove surface particulate matter. A 70- $\mu \mathrm{m}$ thick double-sided tape was used as a spacer, which formed a chamber of dimensions $3 \mathrm{~mm} \times 1.25 \mathrm{~cm} \times$ $70 \mu \mathrm{m}$. The top electrode was placed using a separate 3D-printed holder device (Figure S1). Once the device was constructed using the above protocol, solution was perfused into the chamber using a pipette and a filter paper for suction. We used flat copper electrode clips in a three-electrode configuration to connect to our capacitor device. The counter electrode was connected to the lower electrode, and the working and reference electrodes were connected to the top electrode of our device. 


\subsection{Impedance Measurements}

Experiments were conducted using Electrochemical Impedance Spectroscopy (EIS) on a Zahner Zennium impedance analyser. The parallel-plate contact device was placed into the 3D-printed holder for stabilisation (Figure S1). The contacts from the machine were connected to the parallel-plate device using flat-faced copper alligator clips. A three-electrode configuration was used: The counter electrode was attached to the lower contact of the parallel-plate device, whereas the working electrode was attached to the upper contact with the reference electrode orthogonally clipped onto the clip of the working electrode. Within the Thales Z3.04 environment, the potentiostat mode was ON; the stabilisation delay was set to $1 \mathrm{~s}$, the rest potential drift tolerance was set to $250 \mu \mathrm{V}, \mathrm{V}_{\text {rms }}$ was set to $5 \mathrm{mV}$. Solutions were perfused into the experimental chamber using a micropipette tip at one opening, and a filter paper at the other opening for suction, similar to protocols used for Total Internal Reflection Fluorescence (TIRF) microscopy [31]. The frequency range of the EIS measurement was set from $4 \mathrm{MHz}$ to $1 \mathrm{~Hz}$ and data were subsequently collected.

\subsection{Data Analysis}

MT and tubulin samples were analysed using data from five to seven days of experiments. Each day consisted of three to seven solutions for each concentration being tested, with one frequency sweep per solution. Readings of each sweep were saved as a csv file, and next sample was loaded by solution exchange method. Water was run as the first solution for each day of experiments. BRB80T was run prior to MT solutions, and BRB80C were run prior to the free tubulin containing solutions. MT- and free tubulin-containing solutions were run on separate days, in increasing order of concentration. MATLAB (The Mathworks; Natick, MA, USA) scripts were used for data analysis. Fitting to the real and imaginary components of impedance was performed using the function lsqnonlin. Initial guess values for the MT network resistance and capacitance were $10^{5} \mathrm{~F}$ and $10^{-5} \Omega$, respectively, based on visual inspection of raw data. The initial guess values for the nominal series resistor, $\mathrm{R}_{\mathrm{H}}$, were set at $1.78,0.6$ and $0.4 \Omega$ with tubulin concentrations of $0.222,2.222$ and $22.222 \mu \mathrm{M}$, respectively. The $95 \%$ confidence intervals were determined using the function nlparci. Error propagation was performed assuming no relationship between various days of data collection.

\section{Results}

\subsection{Validation of Parallel-Plate Contact Device to Measure Dielectric Properties of Physiologically Relevant Ionic Solutions}

To determine the differences in the dielectric properties of solution caused by the presence of MTs, we first aimed to create an electrode geometry that would be experimentally robust and easily modelled. We fabricated an FTO-coated parallel-plate contact device (Figure 1a), which allowed EIS using a solution-exchange method.

We started by performing EIS on electrolytes found in the cytosol and observed that the imaginary component of impedance became less negative as a function of applied input frequency (Figure 1b). The total impedance of our system was given by:

$$
Z=r_{C}+r_{S} /\left(1+\left(r_{s} \omega C\right)^{2}\right)+j\left(\omega L_{C}-\left(r_{S}^{2} \omega C\right) /\left(1+\left(r_{S} \omega C\right)^{2}\right)\right)
$$

Here, $Z$ is the impedance, $\omega$ is the angular frequency (given by $2 \pi f$ where $f$ is the input voltage frequency), $C$ is the system capacitance, $L_{c}$ is the cable inductance, $r_{s}$ and $r_{c}$ are the solution and cable resistances respectively. We also observed a decrease in the real component of impedance as a function of input frequency (Figure 1c). Such a trend is expected from Warburg impedance [32,33] and is in accordance with the equation:

$$
Z_{\text {complex }}=\left(\mathrm{A}_{\omega}\right) / \sqrt{ } \omega+\left(\mathrm{A}_{\omega}\right) /(j \sqrt{ } \omega),
$$


Here, $\mathrm{Z}_{\text {complex }}$ is the complex impedance and $\mathrm{A}_{\omega}$ is the Warburg coefficient. Our circuit simplifies to the equation below if we ignore the effect of cable inductance $\omega L_{c}$, at frequencies below $10^{5} \mathrm{~Hz}$ :

$$
Z=r_{c}-j / \omega C,
$$

Our results using various electrolytes emulated previous data [34-36] and validated the experimental setup for further analysis.

a
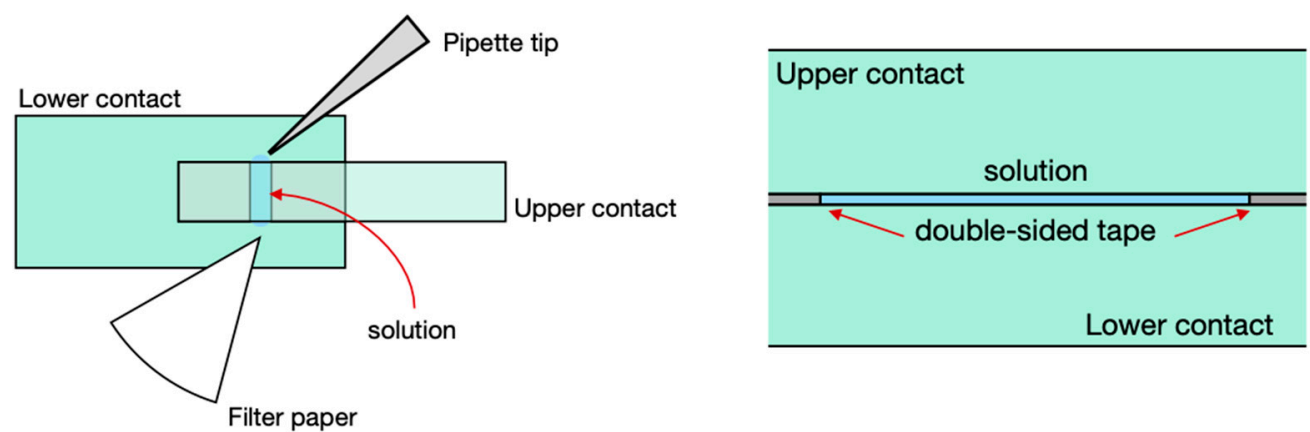

b
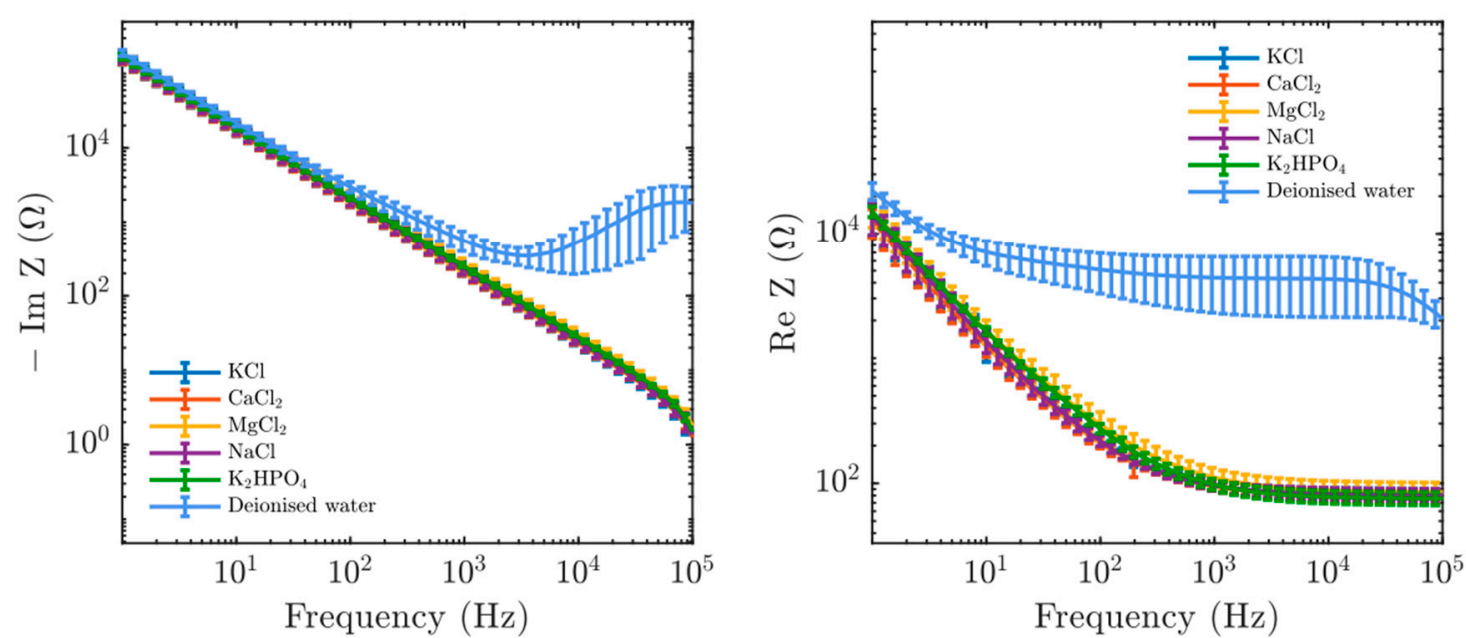

Figure 1. A parallel-plate contact device to measure the impedance properties of microtubules (MTs) compared to tubulin. The operation of the parallel plate device showing (a) top view (left) and side view (right). The upper and lower contacts, double-sided tape and solution are labelled in green, grey and blue, respectively. (b) Imaginary component of impedance for electrolytic solutions at $100 \mathrm{mM}$ and de-ionised water. (c) Real component of impedance for electrolytic solutions at $100 \mathrm{mM}$ and de-ionised water. Data display average values collected between 15 and 21 times. Error bars represent standard deviation.

\subsection{The Effect of Microtubule Networks on Solution Capacitance at Physiologically Relevant Conditions}

We reconstituted and polymerised fluorescent tubulin from a stock of $45.45 \mu \mathrm{M}$ tubulin solution (Materials and Methods). MTs were stabilised using $50 \mu \mathrm{M}$ paclitaxel $[37,38]$ and imaged using an epi-fluorescence microscope. On diluting MT concentration across three orders of magnitude $(0.222$, 2.222 and $22.225 \mu \mathrm{M}$ tubulin), we observed that while individual MTs at low concentrations were separated by large distances, those at cellular concentrations formed enmeshed networks reported previously (Figure 2a-c) [39]. Such interconnected MT networks are utilised by molecular motors 
for long-range macromolecular transport [40,41]. Here, their presence demonstrated successful MT polymerisation for electrical characterisation.

a

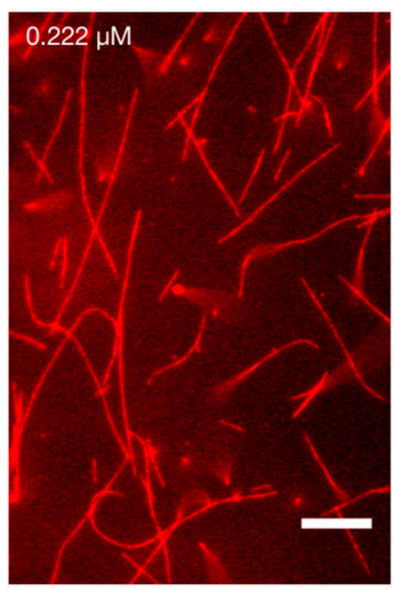

b

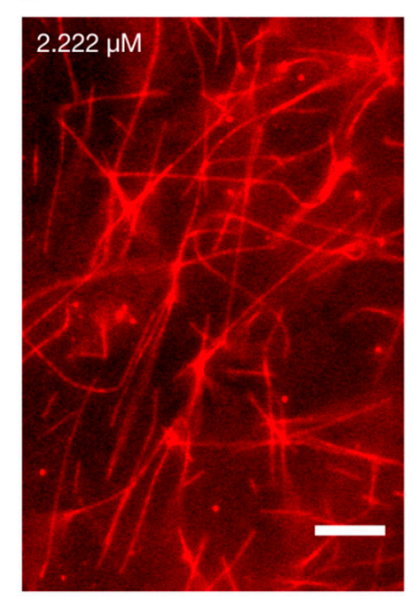

C

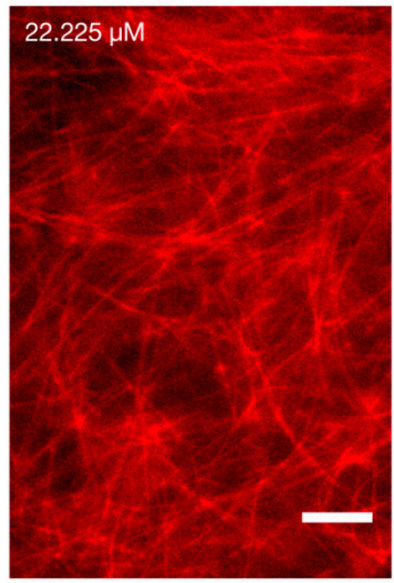

Figure 2. Microtubule imaging at different tubulin concentrations. Polymerisation was performed using $45 \mu \mathrm{M}$ tubulin, and MTs were stabilised with $50 \mu \mathrm{M}$ paclitaxel, and subsequently diluted to a final concentration of (a) $0.222 \mu \mathrm{M}$ tubulin (b) $2.222 \mu \mathrm{M}$ tubulin (c) $22.225 \mu \mathrm{M}$ tubulin, respectively. Scale bars represent $10 \mu \mathrm{m}$.

We performed EIS on BRB80, BRB80T (BRB80 supplemented with $50 \mu \mathrm{M}$ paclitaxel; background for all MT-containing solutions), and MT-containing solutions in increasing order of concentration (Figure 3a,b). We subtracted impedance values obtained for BRB80T alone from those in MT-containing solutions to determine the MT contribution to impedance. Our results showed that with an increasing MT concentration, the value of imaginary impedance became more negative, resulting in positive impedance differences (Figure 3c-f). This effect was greatest at the cell-like $22.225 \mu \mathrm{M}$ tubulin concentration, with impedance differences lowering in magnitude with increasing input frequency (Figure 5a). Experiments with unpolymerised tubulin at the same concentrations were performed using the identical procedure, but using BRB80C (BRB80 was supplemented with $50 \mu \mathrm{M}$ colchicine) as a background, to prevent MT nucleation [42,43]. Results with unpolymerised tubulin did not show an appreciable deviation from zero at any concentration (Figure S5a). The above results suggest that polymerisation of tubulin into MTs alters their ensemble electrical properties, increasing the solution's capacitance on forming MTs and their networks. An increase in the solution's capacitance because of MTs has previously been modelled, $[7,44,45]$ indicating an increase in charge storage as free tubulin polymerises.

\subsection{The Effect of Microtubule Networks on Solution Resistance at Physiologically Relevant Conditions}

Next, we investigated the differences between MTs and tubulin in the real component of impedance (solution resistance). Previous studies using nanomolar tubulin concentrations and low ionic strengths (1-12 mM) have indicated that MTs enhance charge-transport in solutions $[13,20,46]$. To evaluate if this observation held true at physiologically relevant tubulin concentrations and at higher ionic strengths, we also analysed the real component of impedance. Addition of both MTs and tubulin generally led to an increase in solution resistance (Figure 4a-f), with MTs having a higher resistance at low frequencies (1-20 Hz) compared to unpolymerised tubulin. Unexpectedly, a 'reversal' of this behaviour was observed at higher frequencies as MTs began to lower the solution resistance compared to tubulin (Figure 5b). The reversal took place gradually between 10 and $300 \mathrm{~Hz}$ (Figure 6a-d), with a peak between 20 and $60 \mathrm{~Hz}$ (Figure 6e). Interestingly, within this range, we also found that the addition of MTs lowered solution resistance compared to background buffer BRB80T. 
a

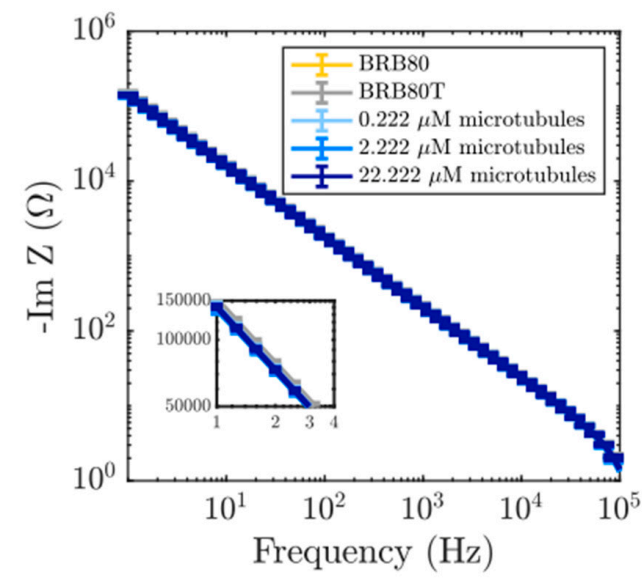

C
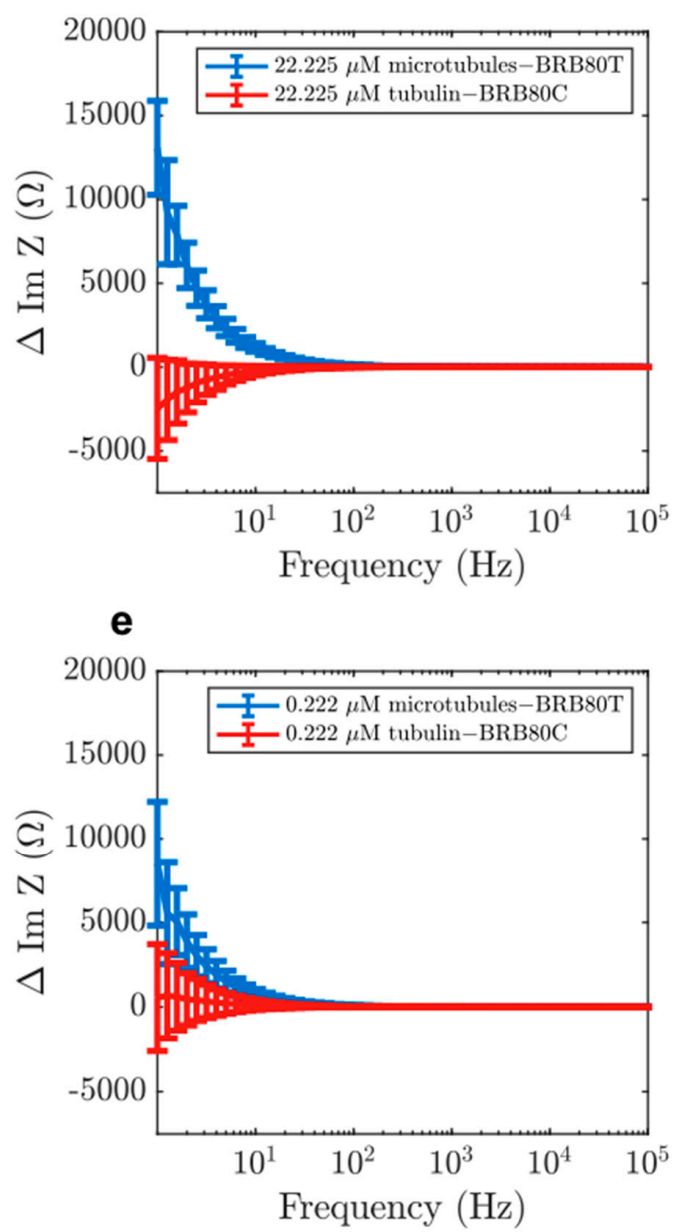

\section{b}

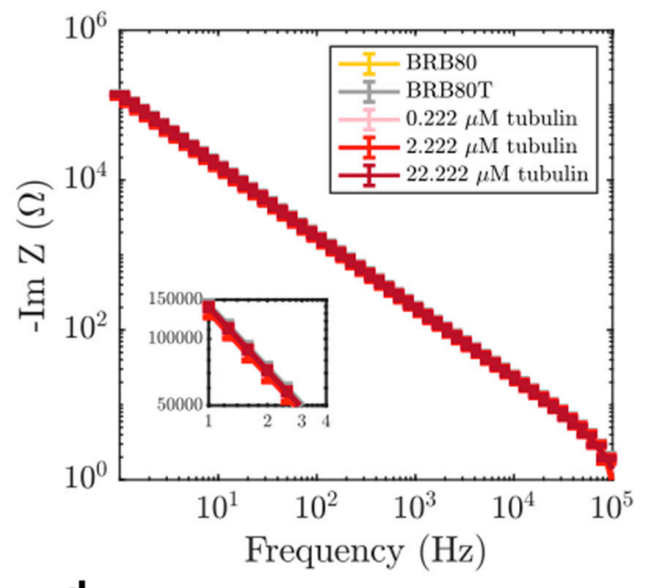

d

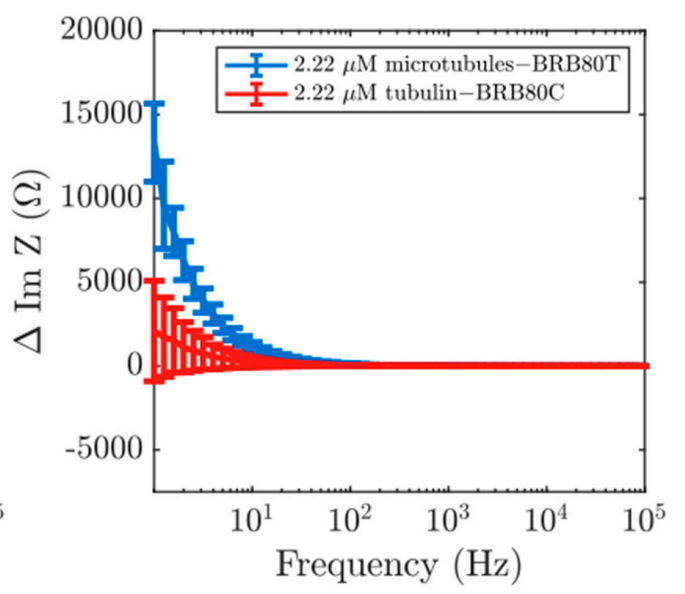

f

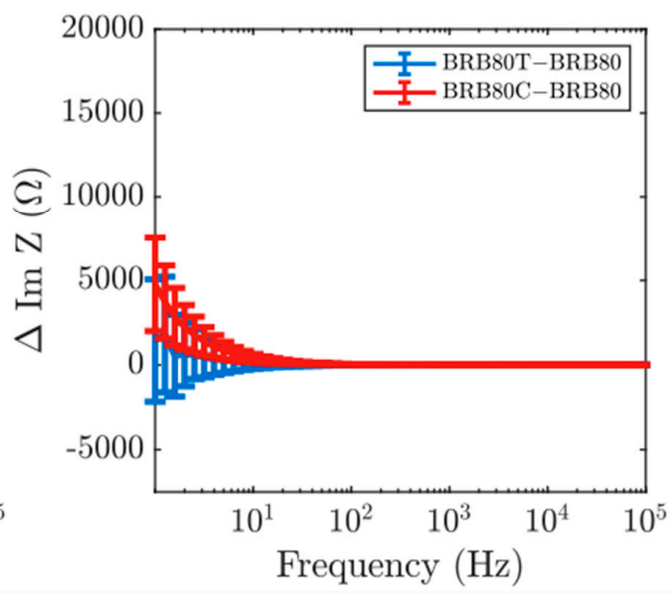

Figure 3. Examples of raw values of imaginary component of impedance in solutions containing (a) MTs and (b) tubulin, for the purpose of displaying typical impedance values. Mean differences in the imaginary component of impedance as a function of input AC (alternating current) frequency at total tubulin concentrations of (c) $22.225 \mu \mathrm{M}$ ( $\mathrm{n}=22$ experiments for tubulin, $\mathrm{n}=21$ for MTs), (d) $2.222 \mu \mathrm{M}$ ( $\mathrm{n}=35$ experiments for tubulin, $\mathrm{n}=49$ for MTs) (e) $0.222 \mu \mathrm{M}(\mathrm{n}=35$ experiments for tubulin, $\mathrm{n}=49$ for MTs), (f) comparison of the effects of paclitaxel (BRB80T) and colchicine (BRB80C, $n=49$ experiments for BRB80T, $\mathrm{n}=35$ for BRB80C, $\mathrm{n}=84$ experiments for BRB80). Error-bars represent standard deviation. 

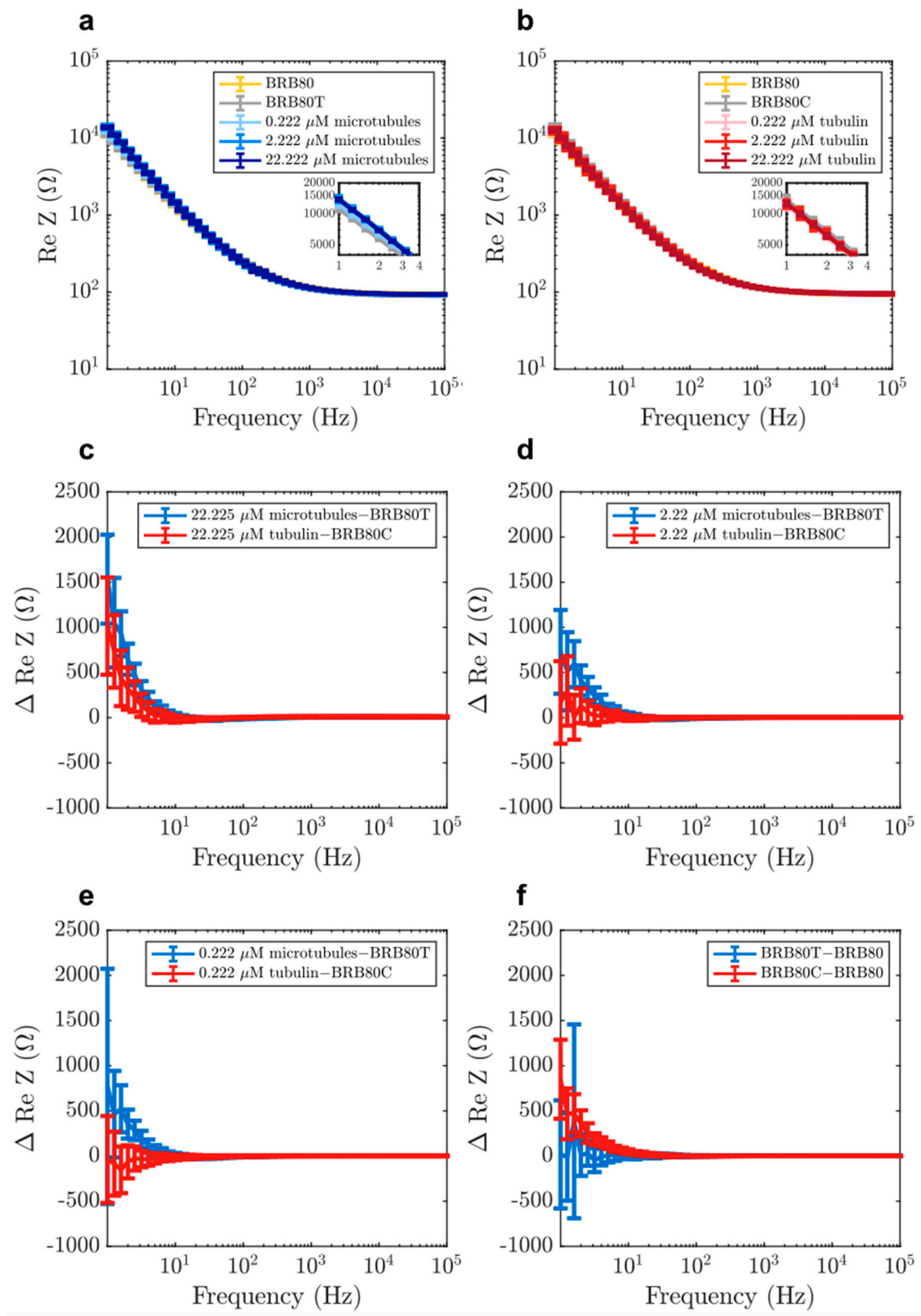

Figure 4. Examples of raw values of real component of impedance in solutions containing (a) MTs and (b) tubulin, for the purpose of displaying typical impedance values. Mean differences in the real component of impedance as a function of input AC frequency at total tubulin concentrations of (c) $22.225 \mu \mathrm{M}(\mathrm{n}=22$ experiments for tubulin, $\mathrm{n}=21$ for MTs), (d) $2.222 \mu \mathrm{M}$ ( $\mathrm{n}=35$ experiments for tubulin, $\mathrm{n}=49$ for MTs) (e) $0.222 \mu \mathrm{M}$ ( $\mathrm{n}=35$ experiments for tubulin, $\mathrm{n}=49$ for MTs), (f) comparison of the effects of paclitaxel (BRB80T) and colchicine (BRB80C, $n=49$ experiments for BRB80T, $n=35$ for $B R B 80 C, n=84$ experiments for BRB80). Error-bars represent standard deviation. 

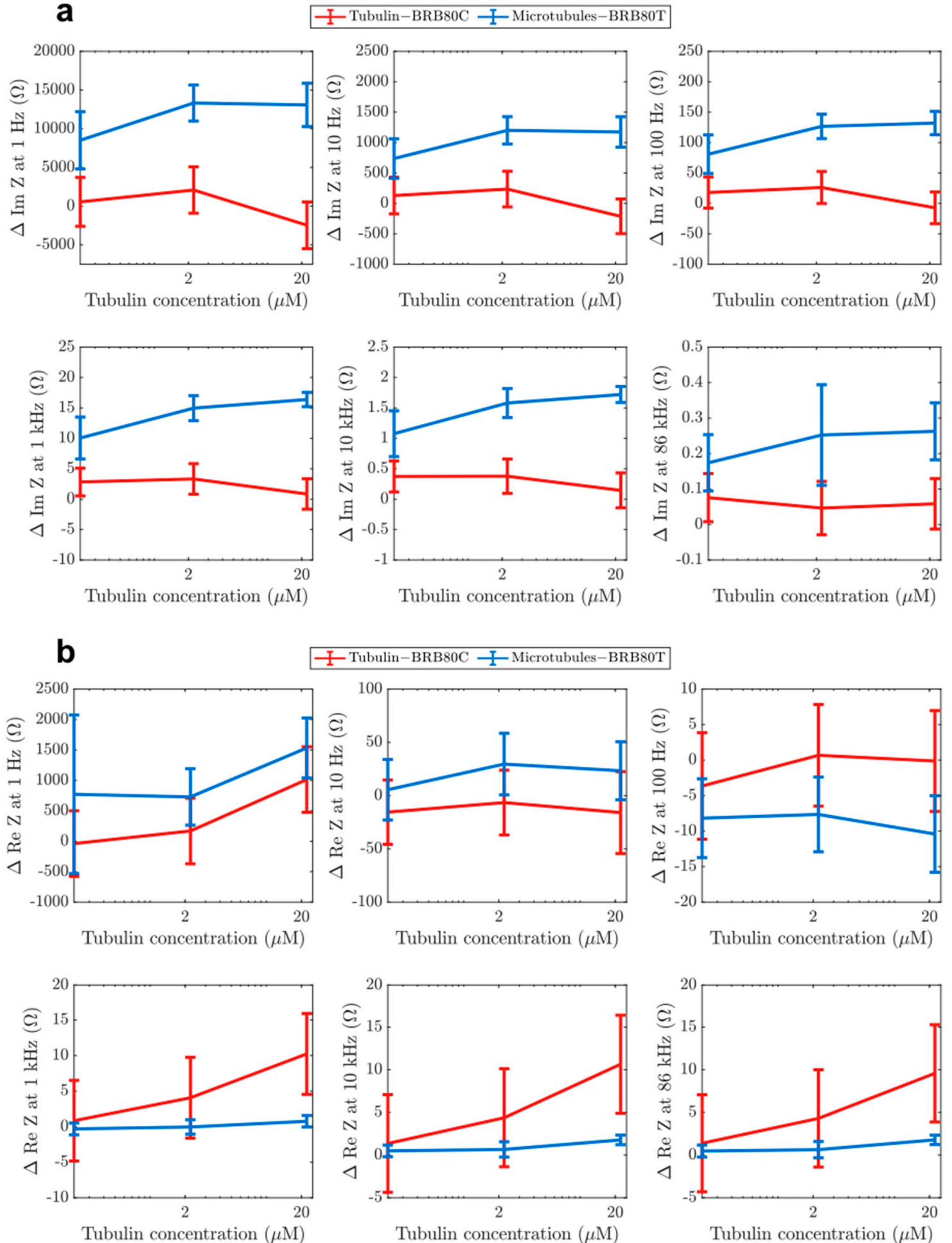

Figure 5. Graphs showing differences in the (a) imaginary from Figure 3 and (b) real component of impedance from Figure 4 as a function of tubulin concentration at input AC frequencies of $1 \mathrm{~Hz}$, $10 \mathrm{~Hz}, 100 \mathrm{~Hz}, 1 \mathrm{kHz}, 10 \mathrm{kHz}$ and $86 \mathrm{kHz}$. Graphs display average values. Error-bars represent standard deviation. 

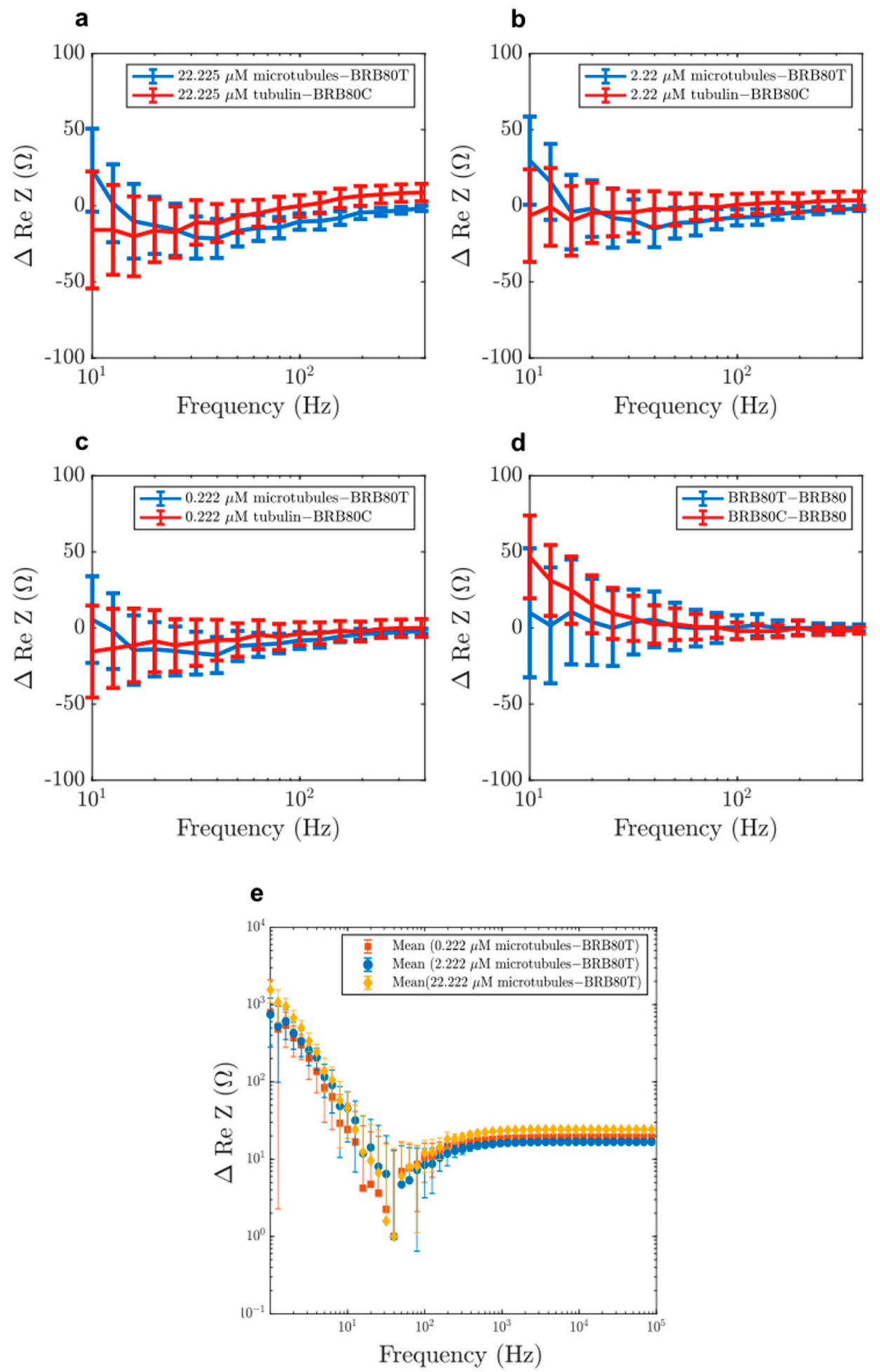

Figure 6. Zoomed in view of the mean differences in the real component of impedance as a function of decreasing input AC frequency at total tubulin concentrations of (a) $22.225 \mu \mathrm{M}$, (b) $2.222 \mu \mathrm{M}$, (c) $0.222 \mu \mathrm{M}$, (d) comparison of the effect of paclitaxel and colchicine on impedance. (e) A logarithmic plot obtained by translating the graphs (a), (b) and (c) upwards. The translation is performed by adding $(1+$ minimum MT solution resistance) to the resistance of each MT concentration. A resistance reversal between $20-60 \mathrm{~Hz}$ is observed, with a peak at $39 \mathrm{~Hz}$ for the $22.225 \mu \mathrm{M}$ concentration. Error-bars represent standard deviation. 
Such a reversal in resistance between microtubules and tubulin has not been reported before. Because the extent of this reversal decreased with decreasing concentration, this result also displays the utility of our 'cell-like' approach. Our results are consistent with predictions of an increase in solution conductance at $\sim 39 \mathrm{~Hz}[9,10]$, which have been hypothesised to arise from oscillatory ionic movement across the MT lattice through nanopores formed between adjacent tubulin dimers (Figure 9a).

It is worth noting that this region falls within the gamma frequency regime $(20-60 \mathrm{~Hz})$, implicating such quasi-resonant phenomena as a possible explanation for the source of low frequency intraneuronal electrical oscillations. No such reversal was observed for the corresponding frequency range in the imaginary impedance values (Figure S4).

\subsection{The Microtubule Network as an RC Circuit in Parallel}

Our next aim is to quantify the resistance and capacitance of the microtubule network. The slope of approximately negative unity on the impedance difference curve suggested that the microtubule network resulted in the addition of a capacitive element to the solution. We examined several combinations but a parallel RC (resistor-capacitor) circuit to represent the entire MT network provided the best fit to observed curves.

We modelled the impedance caused by external circuit elements and BRB80T as $Z_{o}$ and $Z_{s}$ respectively, as shown in Figure 7. The net impedance of the background BRB80T was thus given by:

$$
Z_{\text {buffer }}=Z_{0}+Z_{s}
$$

Denoting the impedance, resistance and capacitance of the entire MT network by $Z_{M T}, R_{M T}$ and $C_{M T}$ respectively, the impedance for the circuit with MTs is given by:

$$
Z_{M T+b u f f e r}=Z_{0}+Z_{S}+R_{H}+Z_{M T}
$$

where,

$$
1 / Z_{M T}=1 / R_{M T}+j \omega C_{M T}
$$

Additionally, the impedance differences between solutions with and without MTs are given by:

$$
\Delta Z=Z_{M T+b u f f e r}-Z_{\text {buffer }}=R_{H}+Z_{M T} \text {, }
$$

where

$$
Z_{M T}=R_{M T} /\left(1+\left(\omega C_{M T} R_{M T}\right)^{2}\right)-j\left(\omega C_{M T} R_{M T}^{2}\right) /\left(1+\left(\omega C_{M T} R_{M T}\right)^{2}\right),
$$

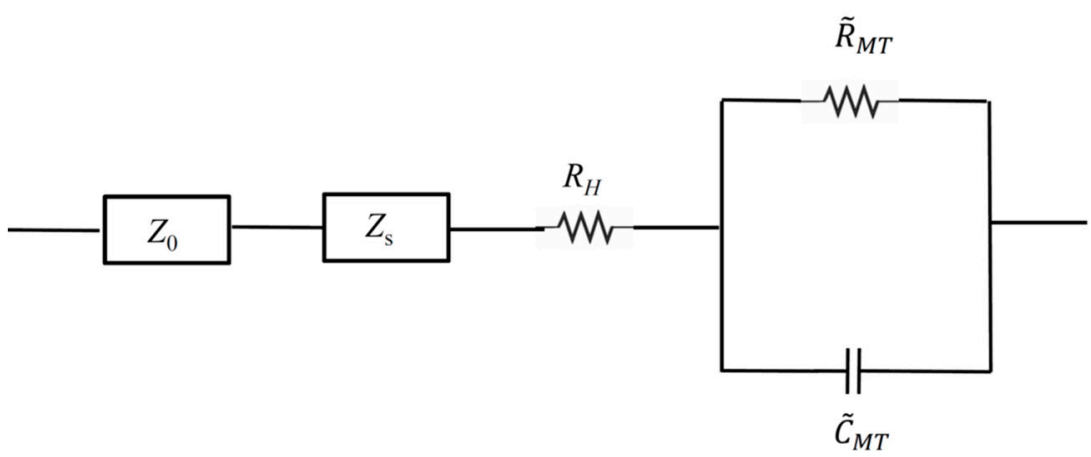

Figure 7. The equivalent electrical circuit model representing the microtubule network as a parallel RC circuit, with network resistance $R_{M T}$ and capacitance $C_{M T}$. The external element has impedance $Z_{0}$, while solution has impedance $Z_{s} . R_{H}$ is the small constant resistance that is ascribed to small fraction of unpolymerised tubulin that is present in MT containing solutions. 
We subsequently fit experimental impedance difference curves shown in Figures 3 and 4 to real and absolute value of imaginary parts of $\Delta Z$ using $R_{H}, R_{M T}$ and $C_{M T}$ as our fit parameters. Here, $R_{H}$ is a resistance ascribed to the nominal fraction of unpolymerised tubulin present in MT containing solutions. The fitted curves are displayed in Figure 8 and the optimal fit parameters are listed in Table 2 (see Materials and Methods for details).
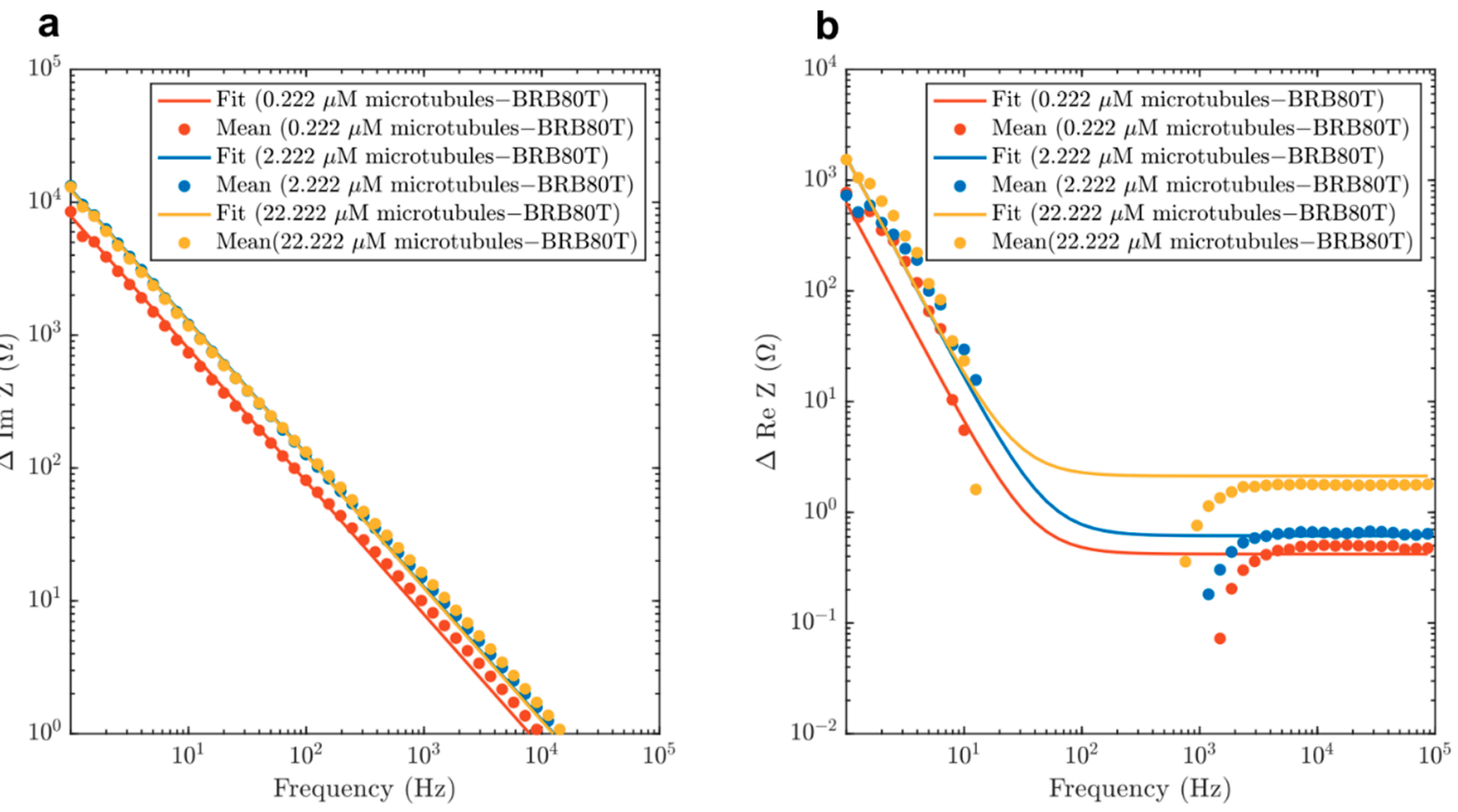

Figure 8. Mean differences of (a) imaginary and (b) real impedance curves for $0.222 \mu \mathrm{M}, 2.222 \mu \mathrm{M}$ and $22.222 \mu \mathrm{M}$, are fitted with the model described in Equation (6) and Figure 7. Fit parameters and confidence intervals are displayed in Table 2. The region between 1-100 Hz was not fit because of the negative differences in resistance from background BRB80T solutions.

Table 2. Fit parameters attained by fitting the real and imaginary components of impedance to Equation (7). Fit parameters represent effective capacitance $C_{M T}$, and resistance $R_{M T}$ introduced into the solution through the addition of the MT network at different concentrations. $R_{H}$ is the small constant resistance that is ascribed to small fraction of unpolymerised tubulin that is present in MT-containing solutions. $\gamma_{R}$ and $\gamma_{C}$ describe the effective resistance and capacitance per unit volume introduced by the microtubule network. $\delta R_{M T}, \delta C_{M T}$ and $\delta R_{H}$ correspond to $95 \%$ confidence intervals for the fit parameters. The values $\delta \gamma_{R}$ and $\delta \gamma_{C}$ correspond to the uncertainties in the resistance and capacitance per unit volume. Corresponding graphs are displayed in Figure 8.

\begin{tabular}{|c|c|c|c|c|c|c|c|c|c|c|}
\hline $\begin{array}{l}{[\mathrm{Tub}]} \\
(\mu \mathrm{M}) .\end{array}$ & $\mathrm{C}_{\mathrm{MT}}(\mathrm{F})$. & $\delta C_{M T}(\mathrm{~F})$ & $R_{M T}(\Omega)$ & $\delta R_{M T}(\Omega)$ & $\begin{array}{l}R_{H} \\
(\Omega)\end{array}$ & $\begin{array}{c}\delta R_{H} \\
(\Omega)\end{array}$ & $\begin{array}{c}\gamma_{R} \\
(\Omega / \mathrm{L})\end{array}$ & $\begin{array}{c}\gamma_{C} \\
(\mathrm{~F} / \mathrm{L})\end{array}$ & $\begin{array}{c}\delta \gamma_{R} \\
(\Omega / \mathrm{L})\end{array}$ & $\begin{array}{l}\delta \gamma_{C} \\
(\mathrm{~F} / \mathrm{L})\end{array}$ \\
\hline 22.222 & $1.27 \times 10^{-5}$ & $1.48 \times 10^{-7}$ & $9.74 \times 10^{4}$ & $1.18 \times 10^{4}$ & 2.12 & 40.61 & $3.71 \times 10^{10}$ & 7.65 & $4.49 \times 10^{9}$ & 0.056 \\
\hline 2.222 & $1.25 \times 10^{-5}$ & $1.67 \times 10^{-7}$ & $1.00 \times 10^{5}$ & $1.40 \times 10^{4}$ & 0.61 & 34.79 & $3.81 \times 10^{10}$ & 4.76 & $5.33 \times 10^{9}$ & 0.063 \\
\hline 0.222 & $2.01 \times 10^{-5}$ & $3.38 \times 10^{-7}$ & $9.97 \times 10^{4}$ & $2.82 \times 10^{4}$ & 0.41 & 31.95 & $3.80 \times 10^{10}$ & 4.83 & $1.07 \times 10^{10}$ & 0.12 \\
\hline
\end{tabular}

\section{Discussion}

Our measurements using a parallel plate contact device reveal interesting electrical properties of MTs at physiological concentrations. Unlike studies exposing MT-containing solutions to non-uniform electric fields [12-14,20], our device allowed robust quantification of electrical impedance in the presence of spatially uniform electric fields. Our results show that the addition of the MT network mimics a parallel RC element placed in series with the high-ionic strength solution, with a nonlinear dependence on MT number. Unpolymerized tubulin did not alter the capacitance significantly, indicating changes in electrical properties of tubulin as it polymerizes. 


\subsection{The Physical Underpinnings of An Increased Capacitance}

An increase in capacitance arises from dense counterion condensation on the MT surface. This has been extensively predicted and simulated to arise from a variety of sources $[7,8,44,47,48]$. First, the negative charge of the tubulin dimer attracts counterions in solution, leading to the presence of a double layer and depletion region outside the microtubule surface $[7,8,46,48]$. The charge distribution in the MT protein wall is also highly non-uniform, with the outer surface containing approximately four times the charge compared to the inner surface [47] (Figure 9c). This asymmetry between the inner and outer electrostatic potentials serves to enhance capacitance and is responsible for the abnormally large dipole moment of the tubulin dimer [1]. The asymmetry also manifests through C-terminal 'tails' composed of 10-12 amino-acids, that can extend 4-5 nm outwards from each tubulin monomer. These slender C-termini tails are highly negative, containing about $50 \%$ of the charge of the tubulin dimer [49]. As they stretch outwards into the solution in a $\mathrm{pH}$ and ionic strength-dependant manner, they increase the effective area of the tubulin dimer and significantly contribute to the overall MT capacitance $[7,8]$.

a

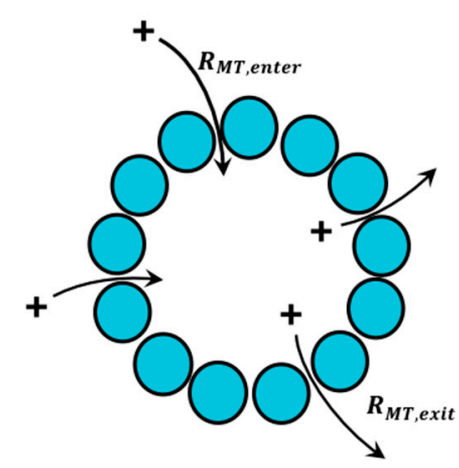

C

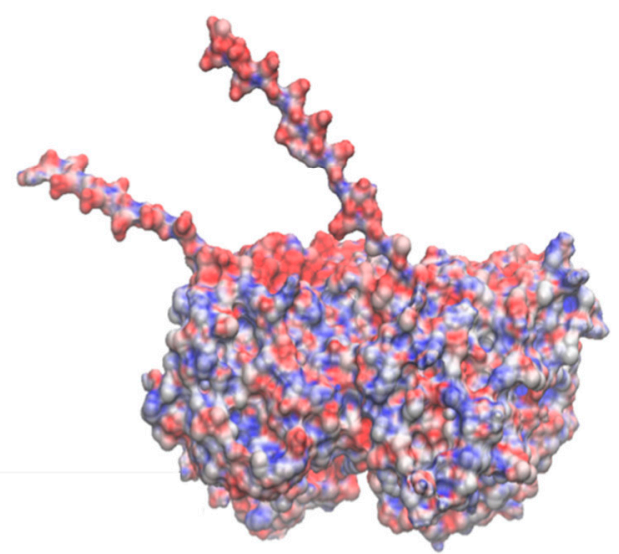

b
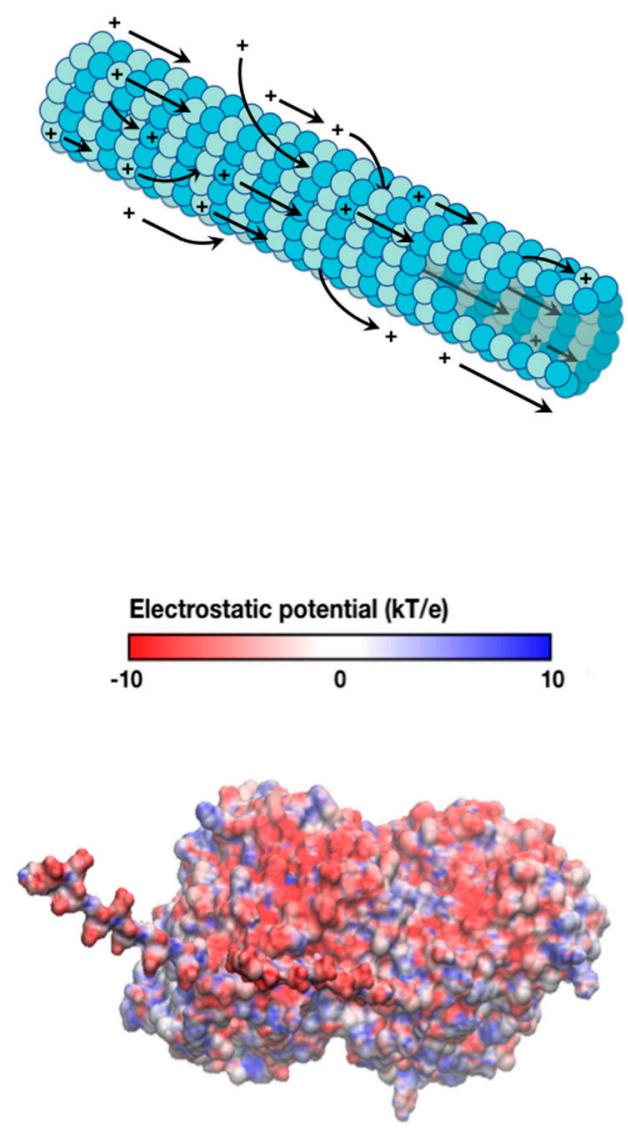

Figure 9. Schematic of charge transport along and across an MT. (a) A representation of charge flow across the MT cross section through nanopores present between adjacent protofilaments. (b) A representation of charge flow through both inner and outer modes along an MT. Arrows depict charge flow via both mechanisms, enabling MT charge storage across a broad spectrum of frequencies, and charge transport at low AC frequencies in the cell. (c) Side view (left) and top view (right) of the tubulin dimer, displaying distribution of electrostatic potential at different locations. The negatively charged C-termini face towards the solution and contains $\sim 50 \%$ of the total negative charge on a tubulin dimer.

Coherent oscillations of these C-terminal tails are modelled to generate solitonic pulses of mobile charge along the outer surface of an MT, creating ionic currents along its length $[7,44,50]$. Ions from the bulk solution are also modelled to be pumped into the hollow MT lumen through nanopores in its 
wall, resulting in charge accumulation inside the cylindrical MT over time [45]. A recent study using molecular dynamics simulations showed that the permeability of the MT lumen was significantly higher for $\mathrm{Na}^{+}$and $\mathrm{K}^{+}$as opposed to $\mathrm{Ca}^{2+}$, allowing for free movement of selective ions into the MT lumen across its porous surface [47]. To the best of our knowledge, our findings are the first to experimentally quantify this resistance encountered by charge flow across the MT cross section. These results implicate not only ionic movement along the microtubule axis, but also across and inside it, enhancing the modelled roles of MTs as complex subcellular nanowires.

Manning's theory of polyelectrolyte solutions predicts the conditions for ionic condensation on charged polymer surfaces provided a sufficiently high linear charge density is present on these surfaces creating an ionic concentration depletion area surrounding them [51]. The sum total of the charges on polymer surfaces and the associated counterions decreases to values dependent on the valence of the counterions and the Bjerrum length, which is the distance from the polymer surface at which the Coulomb energy of the screened surface charges equals the thermal energy. The double layer of surface charges and counterions separated by the Bjerrum length can be viewed as having capacitor-like properties. Although the Manning theory was originally developed for such polyelectrolytes as DNA, it was also applied to actin filaments [52] and MTs [53]. For actin filaments, its application explained the observed lossless transmission of electric pulses along the filament lengths. In the case of MTs, it provided a plausible explanation of unusual amplification of injected electrical signals that propagated along these nanowires. The calculated Bjerrum length for MTs was found to be approximately $6.7 \times 10^{-10} \mathrm{~m}[8,50]$. Both actin filaments and MTs have been represented in these models by cable equations with effective real and imaginary impedance due to the viscosity of the solution-resisting ionic flows and the capacitive properties of the ionic double layers around the filaments, respectively $[52,53]$. The capacitance for a single ring of an MT including C-termini was calculated to be approximately $1.3 \times 10^{-15} \mathrm{~F}$ [8]. When extended to $20 \mu \mathrm{m}$, (representative of the length of a single MT for our measurements), the predicted value would be $\mathrm{C}=3 \times 10^{-12} \mathrm{~F}$, although an experimental confirmation of this prediction is not directly available through our measurements or in any previous work. We note the relatively weak dependence of network capacitance on MT concentration, and assign it to the random spatial locations and directional orientations of MTs in our solution. Indeed, the conductivity of randomly distributed RC networks has been shown to scale weakly with the number of elements in the network [54]. Additionally, qualitative similarities can be found in the models of random resistor and capacitor networks with a frequency-dependent crossover for both conductance and impedance in these networks due to percolation-type conduction [55]. We intend to develop a quantitative model for our experimental observations in a subsequent publication.

\subsection{Implications for the Cell}

Our work, which utilizes cell-like tubulin and ionic concentrations for the first time, indicates a cellular role for microtubules as wires that store charge. Neuronal environments where MTs are spontaneously nucleated from free tubulin, such as growth cones, experience large capacitance changes over short bursts of time. This ability significantly impacts the action potentials that are known to depend strongly on the local charge distributions [56]. Additionally, ionic movement across the MT wall enhances their roles as attenuators of local cation distributions. In nonneuronal environments, transient ionic currents around a MT during mitosis could impact MT dynamics and potentially influence the chromosome segregation. Specifically, $\mathrm{Ca}^{2+}$ ion storage/flow about an MT triggers its depolymerisation, whereas waves of $\mathrm{Mg}^{2+}$ or lowering in the local $\mathrm{pH}$ (increasing $\mathrm{H}^{+}$) leads to MT stabilisation $[57,58]$. The attraction of $\mathrm{Zn}^{2+}$ or $\mathrm{Mn}^{2+}$ ions in the vicinity leads to the formation of two-dimensional tubulin polymers $[59,60]$. Properties of the cytoplasm such as polarisability and relative permittivity get severely attenuated because of the presence of MTs in the vicinity. Because of the polymerisation state of tubulin-altering solution capacitance, our findings implicate a temporal evolution of capacitance and ionic flows as the ratio of MTs to free unpolymerised tubulin changes [61-63]. MT lattice defects, which occur when a tubulin dimer is missing in an MT wall $[64,65]$, cause a large ionic flux to develop at the 
defect site. Spatiotemporal charge distribution shifts are also critical at the MT end, where fluxes form because of sudden changes due to the polymerisation/depolymerisation of the MT. Free/polymerised tubulin hence regulates local and global electrical properties, creating spatially dynamic gradients of charge storage and flux. We envision a cytoskeleton that, in addition to transporting macromolecules, stores and transports ionic signals and electrical information across the cytoplasm (Figure 9a,b).

Our findings can be coupled with a vast array of bio-nanodevices that utilises MTs and MAPs (microtubule-associated proteins) for construction of bio-nanotransporters and bio-actuators [66-70]. Under specific conditions, MAP-MT systems are capable of repositioning macromolecules [71,72], directionally transporting microtubules $[15,73]$ and even drive their movement within zero-mode waveguides [74] and inorganic nanotubes [75]. Storage of electrical charge and its transport along MTs can be coupled to such cutting-edge mechanical MAP-based devices to develop a wide range of nano-actuators and nano-sensors.

When compared to cells, the rates of MT nucleation and polymerisation are significantly lower in BRB80. This difference can be attributed to the absence of MAPs and macromolecular crowding [76,77]. Mammalian cells contain high concentrations of $\mathrm{K}^{+}$ions $(140-300 \mathrm{mM})[27,28]$, which, in addition to MAPs and molecular crowding agents, will be included in a future study to attain physiological equivalence. We also note that the effect of PTMs (post-translational modifications) on the electrical properties of microtubules has not yet been explored. PTMs involve the addition of residues such as phosphate and glutamate that locally influence counterionic condensation around the outer microtubule surface.

We are in the process of performing DC (direct-current) measurements, determine the contribution of MTs to impedance relaxation time and evaluate the voltage dependence of capacitance on MT-containing solutions. Interestingly, this aspect has been discussed previously: the inductance of a single protofilament is calculated to be $<1 \mathrm{fH}$ [8]. Further investigation is required to experimentally confirm these predictions.

\section{Conclusions}

We used EIS to compare the complex impedance of MT- and tubulin-containing solutions. A physiologically relevant, high ionic strength buffer (BRB80) created a high noise, low impedance background, which was countered through the use of physiological concentrations of tubulin. While the presence of MTs increased solution capacitance, unpolymerised tubulin did not have any appreciable effect. In a study that is the first of its kind to the best of our knowledge, we determined the capacitance and resistance of the MT network at physiological tubulin concentrations to be $1.27 \times 10^{-5} \mathrm{~F}$ and $9.74 \times 10^{4} \Omega$. These values correspond to an effective resistance per unit volume of $3.71 \times 10^{10} \Omega / \mathrm{L}$ and effective capacitance per unit volume of $7.65 \mathrm{~F} / \mathrm{L}$. We envision a dual electrical role for MTs in the cell, that of charge storage devices across a broad frequency spectrum (acting as storage locations for ions), and of charge transporters (bionanowires) in the frequency region between 20 and $60 \mathrm{~Hz}$. Our findings also indicate that the electrical properties of tubulin dimers change as they polymerise, revealing the potential impact of MT nucleation and polymerisation on the cellular charge distribution. Our work shows that by storing charge and attenuating local ion distributions, microtubules play a crucial role in governing the bioelectric properties of the cell.

Supplementary Materials: The following are available online at http://www.mdpi.com/2079-4991/10/2/265/s1. Figure S1. 3D printed holders used to fabricate and align the parallel-plate contact device. (a) Top view (left) and (b) side view (right) of holder for the parallel plate device used to perform impedance measurements. (c) Top view (left) and side view (right) of slider used to position the double-sided tape exactly to fabricate the device. (d) Top view (left) and side view (right) of the holder used to position the upper contact precisely on the lower contact. Figure S2. Validation of parallel-plate contact device using $0.5 \mathrm{mM}$ electrolytic solutions. (a) Imaginary component of impedance for electrolytic solutions at $0.5 \mathrm{mM}$ and de-ionised water. (b) Real component of impedance for electrolytic solutions at $100 \mathrm{mM}$ and de-ionised water. Data display average values collected between 15 and 21 times. Error bars represent standard deviation. Figure S3. Example of microtubule and tubulin subtraction with backgrounds to display typical impedance. Figure S4. No 'reversal' in the resistive behaviour of microtubules is observed between 10 and $100 \mathrm{~Hz}$. Graphs showing differences in the real component of impedance as a function 
of decreasing input AC frequency at total tubulin concentrations of (a) $22.225 \mu \mathrm{M}$, (b) $2.222 \mu \mathrm{M},(\mathbf{c}) 0.222 \mu \mathrm{M}$, (d) comparison of the effect of paclitaxel and colchicine on impedance. Figure S5. One-sample t-tests were performed to determine if the impedance difference values were significantly above zero. This was carried out using the t-test function ('ttest') within MATLAB. Graphs showing the variation of obtained p-values for the imaginary components of impedance in (a) tubulin and (b) MT-containing solutions. Graphs showing the variation of obtained p-values for the real components of impedance in (a) tubulin and (b) MT-containing solutions.

Author Contributions: Conceptualization, methodology, software, formal analysis, validation, investigation, data curation, writing-original draft, visualisation, project administration, funding acquisition, A.P.K. Conceptualization, methodology, investigation, validation, writing-original draft, data curation, S.D.P. Methodology, software, formal analysis, validation, investigation, data curation, A.F.B. Conceptualization, investigation, J.P. Software, validation, formal analysis, K.G.S. Methodology, investigation, U.M. Resources, project administration, funding acquisition, J.D.L. Conceptualization, software, formal analysis, validation, investigation, writing - review and editing, visualization, V.R. Conceptualization, methodology, validation, data curation, writing - review and editing, resources, supervision, project administration, funding acquisition, K.S. Conceptualization, methodology, validation, formal analysis, data curation, writing-review and editing, resources, supervision, project administration, funding acquisition, J.A.T. All authors have read and agreed to the published version of the manuscript.

Funding: This research was funded by Novocure LLC, CMC Microsystems, voucher number 6067 and NSERC, grant number 06630. The APC was waived by the publisher for this article.

Acknowledgments: A.P.K. acknowledges Najia Mahdi for helping him with reactive ion etching. A.F.B. acknowledges personnel at The Shack at the University of Alberta and particularly Tristan Stark for teaching him 3D printing. A.F.B. and S.D.P. acknowledge Liam McRae for showing them how to use the impedance analyser. The authors acknowledge Philip Winter, Sheng Zeng, Dayal Pyari Srivastava, Ze'ev Bomzon, Frank Hegmann, Piyush Kar and Kris Carlson for useful discussions.

Conflicts of Interest: The authors declare no conflict of interest. The funders had no role in the design of the study; in the collection, analyses, or interpretation of data; in the writing of the manuscript, or in the decision to publish the results.

\section{References}

1. Mershin, A.; Kolomenski, A.A.; Schuessler, H.A.; Nanopoulos, D.V. Tubulin dipole moment, dielectric constant and quantum behavior: Computer simulations, experimental results and suggestions. Biosystems 2004, 77, 73-85. [CrossRef] [PubMed]

2. Van den Heuvel, M.; De Graaff, M.; Lemay, S.; Dekker, C. Electrophoresis of individual microtubules in microchannels. Proc. Natl. Acad. Sci. USA 2007, 104, 7770-7775. [CrossRef]

3. Timmons, J.J.; Preto, J.; Tuszynski, J.A.; Wong, E.T. Tubulin's response to external electric fields by molecular dynamics simulations. PLoS ONE 2018, 13, e0202141. [CrossRef] [PubMed]

4. Preto, J.; Pettini, M.; Tuszynski, J.A. Possible role of electrodynamic interactions in long-distance biomolecular recognition. Phys. Rev. E 2015, 91, 052710. [CrossRef] [PubMed]

5. Barvitenko, N.; Lawen, A.; Aslam, M.; Pantaleo, A.; Saldanha, C.; Skverchinskaya, E.; Regolini, M.; Tuszynski, J.A. Integration of intracellular signaling: Biological analogues of wires, processors and memories organized by a centrosome 3D reference system. Biosystems 2018, 173, 191-206. [CrossRef]

6. Friesen, D.E.; Craddock, T.J.; Kalra, A.P.; Tuszynski, J.A. Biological wires, communication systems, and implications for disease. Biosystems 2015, 127, 14-27. [CrossRef]

7. Sekulić, D.L.; Satarić, B.M.; Tuszynski, J.A.; Satarić, M.V. Nonlinear ionic pulses along microtubules. Eur. Phys. J. E 2011, 34, 49. [CrossRef]

8. Satarić, M.; Ilić, D.; Ralević, N.; Tuszynski, J.A. A nonlinear model of ionic wave propagation along microtubules. Eur. Biophys. J. 2009, 38, 637-647. [CrossRef]

9. del Rocío Cantero, M.; Etchegoyen, C.V.; Perez, P.L.; Scarinci, N.; Cantiello, H.F. Bundles of Brain Microtubules Generate Electrical Oscillations. Sci. Rep. 2018, 8, 11899. [CrossRef]

10. del Rocío Cantero, M.; Perez, P.L.; Smoler, M.; Etchegoyen, C.V.; Cantiello, H.F. Electrical oscillations in two-dimensional microtubular structures. Sci. Rep. 2016, 6, 27143. [CrossRef] [PubMed]

11. Cifra, M.; Pokorný, J.; Havelka, D.; Kučera, O. Electric field generated by axial longitudinal vibration modes of microtubule. BioSystems 2010, 100, 122-131. [CrossRef]

12. Uppalapati, M.; Huang, Y.M.; Jackson, T.N.; Hancock, W.O. Microtubule alignment and manipulation using AC electrokinetics. Small 2008, 4, 1371-1381. [CrossRef] 
13. Minoura, I.; Muto, E. Dielectric measurement of individual microtubules using the electroorientation method. Biophys. J. 2006, 90, 3739-3748. [CrossRef] [PubMed]

14. Stracke, R.; Böhm, K.; Wollweber, L.; Tuszynski, J.; Unger, E. Analysis of the migration behaviour of single microtubules in electric fields. Biochem. Biophys. Res. Commun. 2002, 293, 602-609. [CrossRef]

15. Isozaki, N.; Ando, S.; Nakahara, T.; Shintaku, H.; Kotera, H.; Meyhöfer, E.; Yokokawa, R. Control of microtubule trajectory within an electric field by altering surface charge density. Sci. Rep. 2015, 5, 7669. [CrossRef] [PubMed]

16. Dujovne, I.; van den Heuvel, M.; Shen, Y.; de Graaff, M.; Dekker, C. Velocity modulation of microtubules in electric fields. Nano Lett. 2008, 8, 4217-4220. [CrossRef] [PubMed]

17. Kirson, E.D.; Gurvich, Z.; Schneiderman, R.; Dekel, E.; Itzhaki, A.; Wasserman, Y.; Schatzberger, R.; Palti, Y. Disruption of cancer cell replication by alternating electric fields. Cancer Res. 2004, 64, 3288-3295. [CrossRef]

18. Giladi, M.; Schneiderman, R.S.; Voloshin, T.; Porat, Y.; Munster, M.; Blat, R.; Sherbo, S.; Bomzon, Z.; Urman, N.; Itzhaki, A. Mitotic spindle disruption by alternating electric fields leads to improper chromosome segregation and mitotic catastrophe in cancer cells. Sci. Rep. 2015, 5, 18046. [CrossRef]

19. Tuszynski, J.; Wenger, C.; Friesen, D.; Preto, J. An overview of sub-cellular mechanisms involved in the action of TTFields. Int. J. Environ. Res. Public Health 2016, 13, 1128. [CrossRef]

20. Santelices, I.B.; Friesen, D.E.; Bell, C.; Hough, C.M.; Xiao, J.; Kalra, A.; Kar, P.; Freedman, H.; Rezania, V.; Lewis, J.D. Response to Alternating Electric Fields of Tubulin Dimers and Microtubule Ensembles in Electrolytic Solutions. Sci. Rep. 2017, 7, 9594. [CrossRef]

21. Shelden, E.; Wadsworth, P. Observation and quantification of individual microtubule behavior in vivo: Microtubule dynamics are cell-type specific. J. Cell Biol. 1993, 120, 935-945. [CrossRef] [PubMed]

22. Van de Water, L.; Olmsted, J. The quantitation of tubulin in neuroblastoma cells by radioimmunoassay. J. Biol. Chem. 1980, 255, 10744-10751. [PubMed]

23. Olmsted, J.; Borisy, G. Ionic and nucleotide requirements for microtubule polymerization in vitro. Biochemistry 1975, 14, 2996-3005. [CrossRef] [PubMed]

24. Weisenberg, R.C. Microtubule formation in vitro in solutions containing low calcium concentrations. Science 1972, 177, 1104-1105. [CrossRef]

25. Böhm, K.J.; Mavromatos, N.E.; Michette, A.; Stracke, R.; Unger, E. Movement and alignment of microtubules in electric fields and electric-dipole-moment estimates. Electromagn. Biol. Med. 2005, 24, 319-330. [CrossRef]

26. Umnov, M.; Palusinski, O.; Deymier, P.; Guzman, R.; Hoying, J.; Barnaby, H.; Yang, Y.; Raghavan, S. Experimental evaluation of electrical conductivity of microtubules. J. Mater. Sci. 2007, 42, 373-378. [CrossRef]

27. van Eunen, K.; Bouwman, J.; Daran-Lapujade, P.; Postmus, J.; Canelas, A.B.; Mensonides, F.I.; Orij, R.; Tuzun, I.; van den Brink, J.; Smits, G.J. Measuring enzyme activities under standardized in vivo-like conditions for systems biology. Febs J. 2010, 277, 749-760. [CrossRef]

28. Theillet, F.-X.; Binolfi, A.; Frembgen-Kesner, T.; Hingorani, K.; Sarkar, M.; Kyne, C.; Li, C.; Crowley, P.B.; Gierasch, L.; Pielak, G.J. Physicochemical properties of cells and their effects on intrinsically disordered proteins (IDPs). Chem. Rev. 2014, 114, 6661-6714. [CrossRef]

29. Fritzsche, W.; Böhm, K.; Unger, E.; Köhler, J.M. Making electrical contact to single molecules. Nanotechnology 1998, 9, 177. [CrossRef]

30. Fritzsche, W.; Köhler, J.M.; Böhm, K.J.; Unger, E.; Wagner, T.; Kirsch, R.; Mertig, M.; Pompe, W. Wiring of metallized microtubules by electron beam-induced structuring. Nanotechnology 1999, 10, 331. [CrossRef]

31. Gell, C.; Bormuth, V.; Brouhard, G.J.; Cohen, D.N.; Diez, S.; Friel, C.T.; Helenius, J.; Nitzsche, B.; Petzold, H.; Ribbe, J. Microtubule dynamics reconstituted in vitro and imaged by single-molecule fluorescence microscopy. In Methods in Cell Biology; Elsevier: New York, NY, USA, 2010; Volume 95, pp. 221-245.

32. Taylor, S.; Gileadi, E. Physical interpretation of the Warburg impedance. Corrosion 1995, 51, 664-671. [CrossRef]

33. Ramos-Barrado, J.; Galan Montenegro, P.; Cambón, C.C. A generalized Warburg impedance for a nonvanishing relaxation process. J. Chem. Phys. 1996, 105, 2813-2815. [CrossRef]

34. Sanabria, H.; Miller, J.H., Jr. Relaxation processes due to the electrode-electrolyte interface in ionic solutions. Phys. Rev. E 2006, 74, 051505. [CrossRef] [PubMed]

35. Lima, L.F.; Vieira, A.L.; Mukai, H.; Andrade, C.M.; Fernandes, P.R. Electric impedance of aqueous $\mathrm{KCl}$ and $\mathrm{NaCl}$ solutions: Salt concentration dependence on components of the equivalent electric circuit. J. Mol. Liq. 2017, 241, 530-539. [CrossRef] 
36. Lenzi, E.; Fernandes, P.; Petrucci, T.; Mukai, H.; Ribeiro, H.; Lenzi, M.; Gonçalves, G. Anomalous diffusion and electrical response of ionic solutions. Int. J. Electrochem. Sci 2013, 8, 2849-2862.

37. Wilson, L.; Miller, H.P.; Farrell, K.W.; Snyder, K.B.; Thompson, W.C.; Purich, D.L. Taxol stabilization of microtubules in vitro: Dynamics of tubulin addition and loss at opposite microtubule ends. Biochemistry 1985, 24, 5254-5262. [CrossRef]

38. Arnal, I.; Wade, R.H. How does taxol stabilize microtubules? Curr. Biol. 1995, 5, 900-908. [CrossRef]

39. Mikhaylova, M.; Cloin, B.M.; Finan, K.; Van Den Berg, R.; Teeuw, J.; Kijanka, M.M.; Sokolowski, M.; Katrukha, E.A.; Maidorn, M.; Opazo, F. Resolving bundled microtubules using anti-tubulin nanobodies. Nat. Commun. 2015, 6, 7933. [CrossRef]

40. Herold, C.; Leduc, C.; Stock, R.; Diez, S.; Schwille, P. Long-Range Transport of Giant Vesicles along Microtubule Networks. ChemPhysChem 2012, 13, 1001-1006. [CrossRef]

41. Kulić, I.M.; Brown, A.E.; Kim, H.; Kural, C.; Blehm, B.; Selvin, P.R.; Nelson, P.C.; Gelfand, V.I. The role of microtubule movement in bidirectional organelle transport. Proc. Natl. Acad. Sci. USA 2008, 105, 10011-10016. [CrossRef]

42. Margolis, R.L.; Wilson, L. Addition of colchicine-tubulin complex to microtubule ends: The mechanism of substoichiometric colchicine poisoning. Proc. Natl. Acad. Sci. USA 1977, 74, 3466-3470. [CrossRef] [PubMed]

43. Olmsted, J.; Borisy, G. Characterization of microtubule assembly in porcine brain extracts by viscometry. Biochemistry 1973, 12, 4282-4289. [CrossRef] [PubMed]

44. Satarić, M.; Sekulić, D.; Živanov, M. Solitonic ionic currents along microtubules. J. Comput. Theor. Nanosci. 2010, 7, 2281-2290. [CrossRef]

45. Freedman, H.; Rezania, V.; Priel, A.; Carpenter, E.; Noskov, S.Y.; Tuszynski, J.A. Model of ionic currents through microtubule nanopores and the lumen. Phys. Rev. E 2010, 81, 051912. [CrossRef] [PubMed]

46. Sanabria, H.; Miller, J.H., Jr.; Mershin, A.; Luduena, R.F.; Kolomenski, A.A.; Schuessler, H.A.; Nanopoulos, D.V. Impedance spectroscopy of $\alpha-\beta$ tubulin heterodimer suspensions. Biophys. J. 2006, 90, 4644-4650. [CrossRef] [PubMed]

47. Shen, C.; Guo, W. Ion Permeability of a Microtubule in Neuron Environment. J. Phys. Chem. Lett. 2018, 9, 2009-2014. [CrossRef]

48. Priel, A.; Tuszyński, J. A nonlinear cable-like model of amplified ionic wave propagation along microtubules. Epl (Europhys. Lett.) 2008, 83, 68004. [CrossRef]

49. Tuszyński, J.; Brown, J.; Crawford, E.; Carpenter, E.; Nip, M.; Dixon, J.; Satarić, M. Molecular dynamics simulations of tubulin structure and calculations of electrostatic properties of microtubules. Math. Comput. Model. 2005, 41, 1055-1070. [CrossRef]

50. Sekulić, D.L.; Satarić, M.V. Microtubule as nanobioelectronic nonlinear circuit. Serb. J. Electr. Eng. 2012, 9, 107-119. [CrossRef]

51. Manning, G.S. Limiting laws and counterion condensation in polyelectrolyte solutions I. Colligative properties. J. Chem. Phys. 1969, 51, 924-933. [CrossRef]

52. Tuszyński, J.; Portet, S.; Dixon, J.; Luxford, C.; Cantiello, H. Ionic wave propagation along actin filaments. Biophys. J. 2004, 86, 1890-1903. [CrossRef]

53. Priel, A.; Ramos, A.J.; Tuszynski, J.A.; Cantiello, H.F. A biopolymer transistor: Electrical amplification by microtubules. Biophys. J. 2006, 90, 4639-4643. [CrossRef] [PubMed]

54. Aouaichia, M.; McCullen, N.; Bowen, C.R.; Almond, D.P.; Budd, C.; Bouamrane, R. Understanding the anomalous frequency responses of composite materials using very large random resistor-capacitor networks. Eur. Phys. J. B 2017, 90, 39. [CrossRef]

55. Panteny, S.; Stevens, R.; Bowen, C. The frequency dependent permittivity and AC conductivity of random electrical networks. Ferroelectrics 2005, 319, 199-208. [CrossRef]

56. Parri, H.R.; Gould, T.M.; Crunelli, V. Spontaneous astrocytic Ca 2+ oscillations in situ drive NMDAR-mediated neuronal excitation. Nat. Neurosci. 2001, 4, 803. [CrossRef]

57. Regula, C.; Berlin, R. Microtubule assembly and disassembly at alkaline pH. J. Cell Biol. 1981, 89, 45-53. [CrossRef]

58. Borisy, G.; Marcum, J.; Olmsted, J.; Murphy, D.; Johnson, K. Purification of tubulin and associated high molecular weight proteins from porcine brain and characterization of microtubule assembly in vitro. Ann. New York Acad. Sci. USA 1975, 253, 107-132. [CrossRef] 
59. Larsson, H.; Wallin, M.; Edström, A. Induction of a sheet polymer of tubulin by Zn2+. Exp. Cell Res. 1976, 100, 104-110. [CrossRef]

60. Nicholson, W.V.; Lee, M.; Downing, K.H.; Nogales, E. Cryo-electron microscopy of GDP-tubulin rings. Cell Biochem. Biophys. 1999, 31, 175-183. [CrossRef]

61. Zhai, Y.; Kronebusch, P.J.; Simon, P.M.; Borisy, G.G. Microtubule dynamics at the G2/M transition: Abrupt breakdown of cytoplasmic microtubules at nuclear envelope breakdown and implications for spindle morphogenesis. J. Cell Biol. 1996, 135, 201-214. [CrossRef]

62. Zhai, Y.; Borisy, G.G. Quantitative determination of the proportion of microtubule polymer present during the mitosis-interphase transition. J. Cell Sci. 1994, 107, 881-890. [PubMed]

63. Kline-Smith, S.L.; Walczak, C.E. Mitotic spindle assembly and chromosome segregation: Refocusing on microtubule dynamics. Mol. Cell 2004, 15, 317-327. [CrossRef] [PubMed]

64. Akhmanova, A.; Dogterom, M. Kinesins lead aging microtubules to catastrophe. Cell 2011, 147, $966-968$. [CrossRef] [PubMed]

65. Bugiel, M.; Mitra, A.; Girardo, S.; Diez, S.; Schäffer, E. Measuring microtubule supertwist and defects by three-dimensional-force-clamp tracking of single kinesin-1 motors. Nano Lett. 2018, 18, 1290-1295. [CrossRef]

66. Diez, S.; Reuther, C.; Dinu, C.; Seidel, R.; Mertig, M.; Pompe, W.; Howard, J. Stretching and transporting DNA molecules using motor proteins. Nano Lett. 2003, 3, 1251-1254. [CrossRef]

67. Hess, H.; Saper, G. Engineering with biomolecular motors. Acc. Chem. Res. 2018, 51, 3015-3022. [CrossRef]

68. Song, W.; Zhu, J.; Kong, W.; Möhwald, H.; Li, J. Different Microtubule Structures Assembled by Kinesin Motors. Langmuir 2018, 34, 9768-9773. [CrossRef]

69. Tas, R.P.; Chen, C.-Y.; Katrukha, E.A.; Vleugel, M.; Kok, M.; Dogterom, M.; Akhmanova, A.; Kapitein, L.C. Guided by Light: Optical Control of Microtubule Gliding Assays. Nano Lett. 2018, 18, 7524-7528. [CrossRef]

70. Fujimoto, K.; Kitamura, M.; Yokokawa, M.; Kanno, I.; Kotera, H.; Yokokawa, R. Colocalization of quantum dots by reactive molecules carried by motor proteins on polarized microtubule arrays. Acs Nano 2012, 7, 447-455. [CrossRef]

71. Adrian, M.; Nijenhuis, W.; Hoogstraaten, R.I.; Willems, J.; Kapitein, L.C. A phytochrome-derived photoswitch for intracellular transport. Acs Synth. Biol. 2017, 6, 1248-1256. [CrossRef]

72. Lam, A.T.-C.; Tsitkov, S.; Zhang, Y.; Hess, H. Reversibly bound kinesin-1 motor proteins propelling microtubules demonstrate dynamic recruitment of active building blocks. Nano Lett. 2018, 18, 1530-1534. [CrossRef] [PubMed]

73. Yokokawa, R.; Takeuchi, S.; Kon, T.; Nishiura, M.; Sutoh, K.; Fujita, H. Unidirectional transport of kinesin-coated beads on microtubules oriented in a microfluidic device. Nano Lett. 2004, 4, 2265-2270. [CrossRef]

74. Fujimoto, K.; Morita, Y.; Iino, R.; Tomishige, M.; Shintaku, H.; Kotera, H.; Yokokawa, R. Simultaneous Observation of Kinesin-Driven Microtubule Motility and Binding of Adenosine Triphosphate Using Linear Zero-Mode Waveguides. Acs Nano 2018, 12, 11975-11985. [CrossRef] [PubMed]

75. Li, J.; Jia, Y.; Dong, W.; Feng, X.; Fei, J.; Li, J. Transporting a Tube in a Tube. Nano Lett. 2014, 14, 6160-6164. [CrossRef]

76. Wieczorek, M.; Chaaban, S.; Brouhard, G.J. Macromolecular crowding pushes catalyzed microtubule growth to near the theoretical limit. Cell. Mol. Bioeng. 2013, 6, 383-392. [CrossRef]

77. Sozański, K.; Ruhnow, F.; Wiśniewska, A.; Tabaka, M.; Diez, S.; Hołyst, R. Small crowders slow down kinesin-1 stepping by hindering motor domain diffusion. Phys. Rev. Lett. 2015, 115, 218102. [CrossRef]

(C) 2020 by the authors. Licensee MDPI, Basel, Switzerland. This article is an open access article distributed under the terms and conditions of the Creative Commons Attribution (CC BY) license (http://creativecommons.org/licenses/by/4.0/). 